\title{
Long non-coding RNA SNHG3 promotes the progression of clear cell renal cell carcinoma via regulating BIRC5 expression
}

\author{
Zhaoyu Xu, Junjie Ye, Pengfei Bao, Qi Wu, Fuchen Xie, Peng Li \\ Department of Urology, Lishui People's Hospital, Lishui, China \\ Contributions: (I) Conception and design: P Li; (II) Administrative support: F Xie; (III) Provision of study materials or patients: Q Wu; (IV) \\ Collection and assembly of data: P Bao; (V) Data analysis and interpretation: J Ye, Z Xu; (VI) Manuscript writing: All authors; (VII) Final approval of \\ manuscript: All authors. \\ Correspondence to: Peng Li, MD. Department of Urology, Lishui People's Hospital, No. 15, Mass Street, Liandu District, Lishui 323000, China. \\ Email: 18957091072@189.cn.
}

\begin{abstract}
Background: Research has shown that the progression of clear cell renal cell carcinoma (ccRCC) is modulated by long non-coding RNAs (lncRNAs). However, the roles of specific lncRNAs in the malignancy of ccRCC are still unknown.

Methods: TCGA and GSE66272 datasets were used to predict differentially expressed genes (DEGs) in ccRCC. ENCORI database was employed to display BIRC5 miRNA network and potential lncRNA interactions for miRNAs. KM plotter and correlation analyses were performed to identify the overall survival (OS)- and BIRC5-related miRNAs. Quantitative real-time PCR (qRT-PCR) was used to verify the BIRC5 mRNA in the seventy paired clinical samples of ccRCC tissues. The ccRCC A498 and 786-O were individually transfected with lncRNA SNHG3 and LINC00997 and then western blotting was used to detect the BIRC5 protein expression. The Dual-luciferase reporter assay was used to examine the regulatory interaction between lncRNA SNHG3 and microRNA (miRNA/miR)-10b-5p.

Results: BICR5 is associated with the progression of ccRCC. The two novel lncRNAs (LINC00997, SNHG3) were up-regulated in ccRCC tissues and positively with the BICR5 protein expression. However, Suppressing SNHG3 expression reduced BIRC5 protein expression compared with the LINC00997, most importantly, Suppressing SNHG3 expression suppressed tumor progression in vitro. In addition, SNHG3 promotes the expression of BIRC5 protein by sponging microRNA-10b-5p.

Conclusions: Our findings suggest that SNHG3 plays a vital role in promoting ccRCC via the microRNA10b-5p/BIRC5 axis and may serve as a novel therapeutic target for the treatment of patients with ccRCC.
\end{abstract}

Keywords: LncRNA SNHG3; miR-10b-5p; BIRC5; clear cell renal cell carcinoma (ccRCC)

Submitted Aug 17, 2021. Accepted for publication Sep 29, 2021.

doi: $10.21037 /$ tcr-21-1802

View this article at: https://dx.doi.org/10.21037/tcr-21-1802

\section{Introduction}

Renal cell carcinoma (RCC) is one of the world's most common malignancies, accounting for $4.2 \%$ of all new cancer diagnoses (1). Clear cell renal cell carcinoma (ccRCC) makes up $75 \%$ of all kidney cancers (2). Due to the resistance of ccRCC to radiotherapy and chemotherapy, surgery is the principle treatment for the disease (3). Although most patients receive early surgical treatment, ccRCC recurs in $30 \%$ of cases (4). Therefore, novel biomarkers and targeted therapies are urgently needed to improve the outcomes of patients with ccRCC.

Long non-coding RNAs (lncRNAs) are defined as RNA molecules longer than 200 nucleotides which have little capacity for coding protein (5). Emerging evidence has confirmed that lncRNAs can modulate gene epigenetic, transcriptional, and post-transcriptional processing, which promote disease progression, especially in cancer (6). In ccRCC, lncRNAs are differentially expressed and exert 
an important role in promoting metastasis. For instance, the lncRNA SNHG3 is highly expressed in ccRCC and promotes the proliferation and migration of ccRCC cells (7), while LINC00997 contributes to ccRCC metastasis and is a potential prognostic biomarker (8). Therefore, it is vital to investigate the role of differential lncRNAs in promoting ccRCC metastasis and the underlying mechanism.

In this study, we firstly used the Gene Expression Omnibus (GEO) dataset GSE12470 and The Cancer Genome Atlas (TCGA) to investigate differentially expressed genes (DEGs) in ccRCC. The baculoviral inhibitor of apoptosis repeatcontaining 5 (BIRC5) gene was found to be highly expressed in CCRCC tissues. Subsequently, the upstream lncRNAs of BIRC5 were predicted. Through expression correlation and survival analyses, two potential lncRNAs (SNHG3 and LINC00997) were identified as regulators of BIRC5 expression. Finally, we explored the potential upstream dysregulated mechanisms and modulatory pathways of BIRC5.

We present the following article in accordance with the MDAR reporting checklist (available at https://dx.doi. org/10.21037/tcr-21-1802).

\section{Methods}

\section{Clinical samples}

Seventy paired clinical samples of ccRCC tissues and neighboring normal tissues were acquired from patients treated in the Department of Urology at Lishui People's Hospital (Zhejiang, China). The study was conducted in accordance with the Declaration of Helsinki (as revised in 2013). The study was approved by the Ethics Committee of Lishui People's Hospital (No.: LLW-F0-403) and informed consent was taken from all the patients.

\section{Cell culture}

The renal cancer cell lines A498 and 786-O and the normal kidney epithelial cell line HK2 were obtained from the American Type Culture Collection (ATCC). The cells were cultured in Dulbecco's Modified Eagle Medium (DMEM; Invitrogen, Carlsbad, CA, USA) containing 1\% penicillin/ streptomycin (Invitrogen, Carlsbad, CA, USA) and 10\% fetal bovine serum (FBS; Gibco) in a $37{ }^{\circ} \mathrm{C}$ incubator containing $5 \% \mathrm{CO}_{2}$.

\section{Quantitative real-time PCR}

Total RNA was extracted from cells and tissues using
TRIzol (Invitrogen, Carlsbad, CA, USA) in adherence with the manufacturer's instruction. The primer for BIRC5 was acquired from Invitrogen Bioengineering Corporation (Shanghai, China). Quantitative real-time PCR (qRTPCR) was performed as previously described (9). The $2^{-\Delta \Delta C t}$ method was used to estimate the relative RNA expression levels. Each sample was assayed in triplicate.

\section{Transwell migration and invasion assays}

Transwell assay was employed to examine cell invasion. Briefly, each Transwell chamber was precoated with $80 \mu \mathrm{L}$ Matrigel. Then, cells $\left(3 \times 10^{5}\right)$ in $400 \mu \mathrm{L}$ of serum-free DMEM were seeded into the upper chamber, and $400 \mu \mathrm{L}$ DMEM with $10 \%$ serum was added to the lower chamber. After 24-hour culture at $37^{\circ} \mathrm{C}$, the invading A498 and 786-O cells were fixed and stained with $0.5 \%$ crystal violet (Beyotime Institute of Biotechnology, Beijing, China). Finally, photomicrographs were taken under an inverted light microscope (Leica, Germany).

\section{Construction of cell lines stably transfected with lncRNA SNHG3 and LINC00997}

The well-designed small interfering RNA (siRNA) oligos of lncRNA SNHG3 and LINC00997, along with their corresponding control siRNAs, were generated by Shanghai Gene Tech (Shanghai, China), and then separately transfected into cells with RNAiMAX (Invitrogen, USA) in Opti-MEM (Invitrogen, USA) for 48 hours following the manufacturer's protocol. The transfection efficiency was evaluated by green fluorescent protein assay, and stable cell lines were screened out with puromycin.

\section{Wound-healing assay}

Cell migration was measured by wound healing assay. Briefly, A498 and 786-O cells were seeded on 6-well plates and cultured to $90 \%$ confluence. Then, a scrape was made using a $200-\mu \mathrm{L}$ pipette tip. At 0 and 48 hours after scratching, images were captured under an inverted light microscope (Leica, Germany).

\section{Western blotting}

The cellular proteins of A498 and 786-O cells in each group were collected using RIPA lysis buffer (Beyotime Institute of Biotechnology, Beijing, China). The protein 
concentrations were quantified by bicinchoninic acid assay. Then, equal amounts $(50 \mu \mathrm{g})$ of protein were separated by $15 \%$ SDS-PAGE (sodium dodecyl sulfate-polyacrylamide gel electrophoresis) and transferred onto polyvinylidene difluoride membranes. The membranes were blocked with $5 \%$ non-fat milk in tris-buffered saline with Tween 20 for 1 hour at room temperature and then incubated with antiBIRC5 antibody (1:1,000, Abcam, USA) overnight at $4{ }^{\circ} \mathrm{C}$. The next day, after washing, the membranes were incubated with horseradish peroxidase (HRP)-conjugated secondary anti-rabbit $\operatorname{IgG}$ (1:2,000, Beyotime Institute of Biotechnology, Beijing, China) for 1 hour at room temperature. Finally, the membranes were imaged using the Bio-Rad imaging system (ImageLab ${ }^{\mathrm{TM}}$ software version 2.0.0.27).

\section{Dual-luciferase reporter assay}

Dual-luciferase reporter assay was used to examine the regulatory interaction between lncRNA SNHG3 and microRNA (miRNA/miR)-10b-5p. Plasmids based on the dual-luciferase reporter vector of SNHG3-WT (wildtype) and SNHG3-MT (mutant-type) were constructed and confirmed through DNA sequencing (Shanghai GenePharma Co., Ltd, Shanghai, China). The plasmids were then transfected into A498 and 786-O cells with miR10b-5p mimics or miRNA negative control (miR-NC) using Lipofectamine 2000 reagent (Invitrogen, Thermo Fisher Scientific, USA) in adherence with the manufacturer's protocol. The transfected cells were cultured for a further 48 hours, after which the luciferase reporter assay was executed as previously described (10). Experimental results were analyzed in triplicate and normalized by Renilla luciferase activity.

\section{Data collection}

The GSE66272 dataset was obtained from the GEO database (https://www.ncbi.nlm.nih.gov/geo/). The miRNA-seq and mRNA-seq data and corresponding clinicopathological information of patients with ccRCC in the TCGA database were downloaded from the GDC (Genomic Data Commons) database (https://portal.gdc.cancer.gov/). All data were normalized before further analysis was conducted. Differential expression analysis was performed in $\mathrm{R} . \mathrm{P}<0.05$ was considered statistically significant.

\section{ENCORI database}

The ENCORI (Encyclopedia of RNA Interactomes) database is a robust open-source platform mainly focused on non-coding RNAs and their potential target interactions. The database can be used to analyze miRNAncRNA, miRNA-mRNA, ncRNA-RNA, and RNA-RNA interactions from CLIP-seq, degradome-seq, and RNARNA interactome data (11). For the present work, the ENCORI database was utilized to uncover the potential competing endogenous RNA (ceRNA) network of BIRC5. All microRNAs and lncRNAs associated with BIRC5 were downloaded for further analysis in patients with ccRCC.

\section{Correlation analysis}

The correlations among lncRNAs, miRNAs, and BIRC5 were analyzed to identify the specific regulatory ceRNA network of BIRC5. $\mathrm{P}<0.05$ was considered statistically significant. The specific parameters are all shown inside the corresponding figures.

\section{Survival analysis}

The correlations between DEG expression and survival in patients with ccRCC was analyzed in R. A Cox P value of $<0.05$ was considered statistically significant. To investigate the relationship of prognosis-related mRNAs, microRNAs, and lncRNAs with survival of ccRCC, overall survival (OS) and Kaplan-Meier (KM) plotter analyses were conducted in patients with ccRCC from TCGA.

\section{Establishment of a lncRNA-microRNA-BIRC5 regulatory network}

The whole microRNA-BIRC5 regulatory network and IncRNA-microRNA-BIRC5 ceRNA network for BIRC5 were constructed with Cytoscape Java version 3.7.16 software (12).

\section{Key patbways related to the prognosis of ccRCCs}

The Key pathways related to the prognosis of ccRCCs were analyzed by GO and KEGG enrichment. The enrichment of GO and KEGG was expressed with a bubble plot and rectangle showing the gene ratio, gene count, gene function description, and $\mathrm{P}$ value.

\section{Statistical analysis}

Differential expression analysis between tumor and 

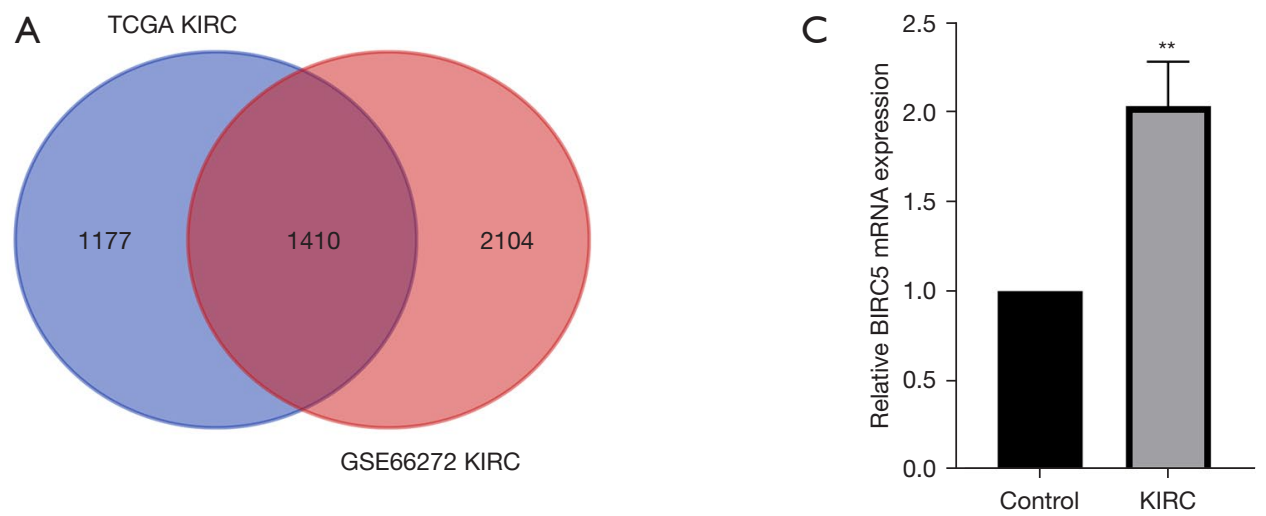

B Type 官Normal 追Tumor

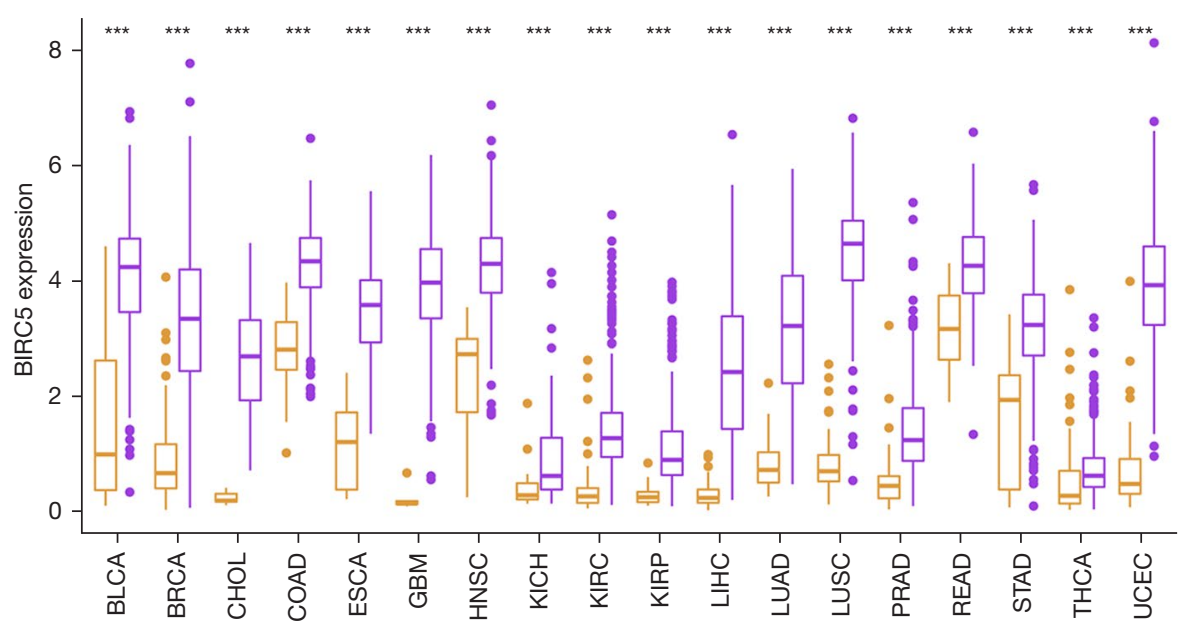

Figure 1 BICR5 is associated with the progression of ccRCC. (A) Comparison of DEGs in patients with ccRCC between the TCGA and GSE66272 datasets. (B) Expression characteristics of BIRC5 across 33 cancer types in TCGA database. (C) mRNA expression of BIRC5 in ccRCC cell in 70 paired normal and tumor tissues in our center. ${ }^{* *}, \mathrm{P}<0.01$; ${ }^{* *}, \mathrm{P}<0.001$. ccRCC, clear cell renal cell carcinoma; DEG, differentially expressed gene; TGCA, The Cancer Genome Atlas.

adjacent normal tissues from patients with ccRCC were calculated using Student's two-tailed $t$-test, with asterisks used to denote genes with differential expression. $\mathrm{P}$ values of less than $0.05,0.01,0.001$, or 0.0001 were considered statistically significant and are indicated by “*”, “**», “***”, and “****”, respectively.

\section{Results}

\section{BICR5 is associated with the progression of ccRCC}

To obtain the robust DEGs between adjacent and tumor tissues from patients with ccRCC, two ccRCC datasets were subjected to limma analysis. The GSE66272 dataset consists of 26 normal and 26 tumor tissues, and the TCGA dataset was composed of 72 normal and 535 tumor tissues from patients with ccRCC. As shown in Figure 1A, there were 2,587 and 3,514 DEGs obtained from the TCGA and GSE66272 datasets, respectively. There were 1,410 overlapping genes between the two datasets, which were obtained for further analysis.

$\mathrm{KM}$ plotter and univariate Cox analyses were performed to filter out the high-risk genes in patients with ccRCC (available online: https://cdn.amegroups.cn/static/public/ tcr-21-1802-01.pdf). A total of 1,056 high-risk genes were 
collected, and these exhibited significant differences between adjacent and tumor tissues. Considering that some oncogenes can exert their function across multiple cancers, the RNA sequencing data of 33 cancers in TCGA were collected, and pan-cancer analysis of these 1,056 high-risk genes was performed. Notably, BIRC5 was significantly up-regulated across all 33 malignancies (Figure 1B). More importantly, we detected higher BICR5 mRNA expression in ccRCC tissues than in the neighboring normal tissues (Figure 1C). To sum up, these results highlight the crucial role of BIRC5 as a tumor promoter gene in malignancies, especially in ccRCC.

\section{Construction of a miRNA network for BIRC5 and key pathways related to the prognosis of ccRCC}

Previous research has demonstrated that BIRC5 can exert oncogenic functions in other malignancies $(13,14)$, which prompted us to investigate its role in the progression and development of ccRCC. Given that various studies have evidenced the vital involvement of ceRNAs in tumor development, the subsequent analysis was focused on constructing the ceRNA network for BIRC5. Initially, 200 miRNAs that might target BIRC5 were collected from the ENCORI database (11). Then, the miRNA sequencing data of patients with ccRCC were downloaded from the TCGA database, and the expression levels of the 200 predicted miRNAs were extracted for further analysis. The BIRC5 miRNA network is displayed in Figure $2 \mathrm{~A}$. Then, we conducted GO and KEGG enrichment analyses for the prognosis-related DEGs. Results show that most DEGs play crucial roles in cell cycle, nuclear division, TGFbeta signaling pathway and so on, which have been widely reported in promoting tumor progression (Figure 2B).

\section{Four microRNAs (miR-101-3p, miR-10b-5p, $m i R-27 b-3 p$, and miR-10a-5p) are negatively correlated with BICR5 gene expression}

To further investigate the miRNAs which are both negatively correlated with BIRC5 and associated with OS in ccRCC, KM plotter and correlation analyses were performed to identify the OS- and BIRC5-related miRNAs among the 200 predicted miRNAs. Four robust miRNAs met these stringent criteria: miR-101-3p, miR-10b-5p, miR-27b-3p, and miR-10a-5p (Figure 3A,3B). As shown in Figure $3 A$, these four miRNAs were negatively correlated with BIRC5, which indicated that they might be able to regulate BIRC5 expression. Furthermore, the up-regulation of these four miRNAs was related to a better prognosis (Figure 3B), suggesting that they might function as tumor suppressors in ccRCC.

\section{The constructed lncRNA-miRNA-BIRC5 network is associated with ccRCC progression}

Given that the four miRNAs had negative relationships with BIRC5 in ccRCC, we next constructed the ceRNA network of BIRC5. The potential lncRNA interactions for the four miRNAs were also collected from the ENCORI database (11). There were 77, 78, 153, and $63 \mathrm{lncRNAs}$ potential regulators predicted for hsa-miR-10a-5p, hsa-miR-10b-5p, hsa-miR27b-3p, and hsa-miR-101-3p, respectively. All these potential lncRNAs were collected for correlation and OS analyses to identify key lncRNAs which, in addition to being promoters of ccRCC progression, were negatively correlated with the four miRNAs and positively correlated with BIRC5 expression.

Only two lncRNAs were screened out: SNHG3 and LINC00997. As shown in Figure 4A,4B, we found that SNHG3 had a positive correlation with BIRC5 $(\mathrm{R}=0.21$, $\mathrm{P}<0.05)$ but had a negative correlation with hsa-miR-10b$5 \mathrm{p}(\mathrm{R}=-0.26, \mathrm{P}<0.05)$, showing the underlying ceRNA network between them. Moreover, SNHG3 was significantly increased in tumor tissues compared to adjacent normal tissues (Figure 4C). LINC00997 was positively correlated with BIRC5 $(\mathrm{R}=0.29, \mathrm{P}<0.05)$ and negatively correlated with hsa-miR-27b-3p $(\mathrm{R}=-0.25, \mathrm{P}<0.05)$, demonstrating another potential ceRNA network related to BIRC5 (Figure 4D,4E). Like SNHG3, LINC00997 was up-regulated in the tumor tissues of patients with ccRCC (Figure 4F).

Subsequently, an OS analysis of SNHG3 and LINC00997 was performed to determine the potential value of these lncRNAs as treatment targets. The results robustly showed that a high expression of SNHG3 or LINC00997 could lead to a poor prognosis (Figure 4G,4H). A network related to BIRC5 (Figure 4I), consisting of two ceRNA networks (the LINC00997/hsa-miR-27b-3p/ BIRC5 axis and the SNHG3/hsa-miR-10b-5p/BIRC5 axis), was also constructed. This network might explain why and how BIRC5 and its ceRNA networks contribute to the carcinogenesis of ccRCC and may provide potential targets for ccRCC treatment.

\section{The lncRNA SNHG3/hsa-miR-10b-5p/BIRC5 axis is a vital pathway contributing to ccRCC progression}

To confirm whether SNHG3 or LINC00997 can regulate 
A

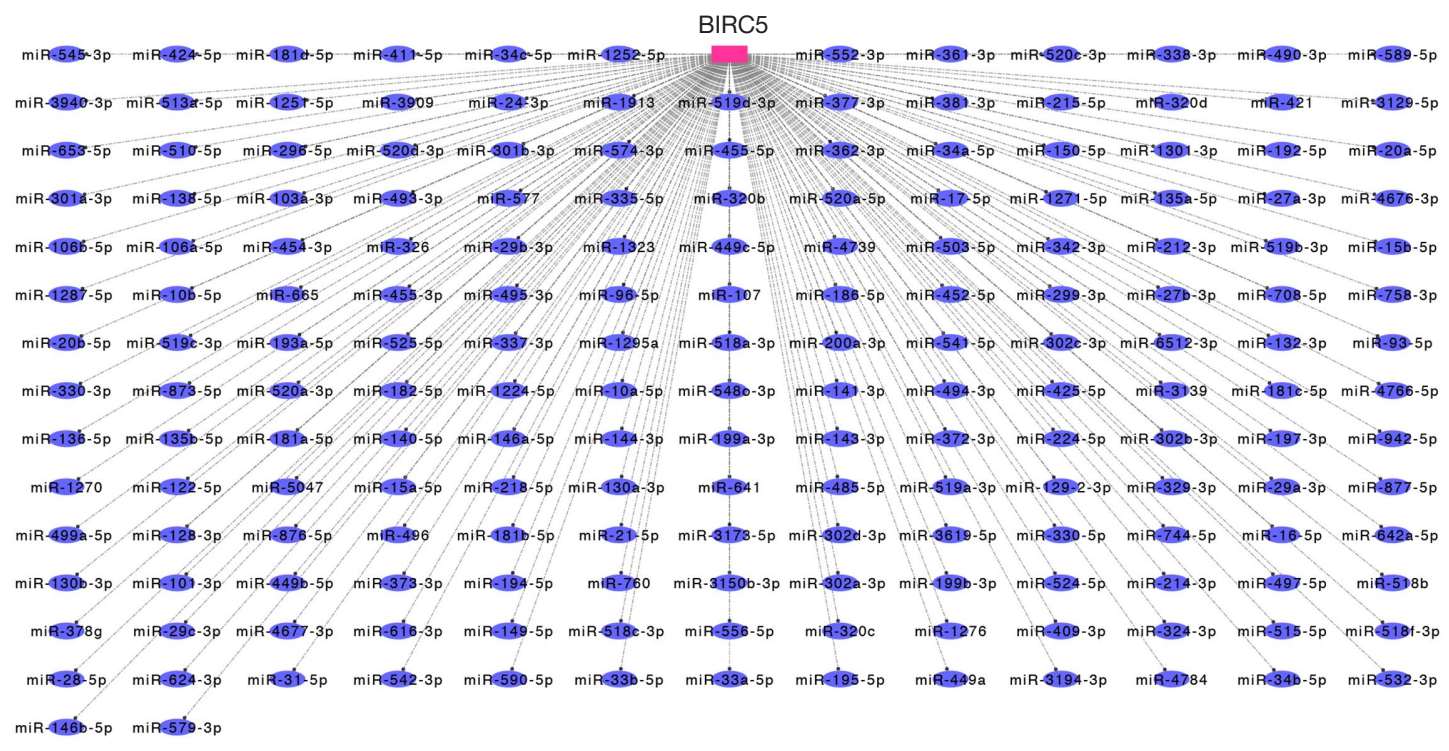

B
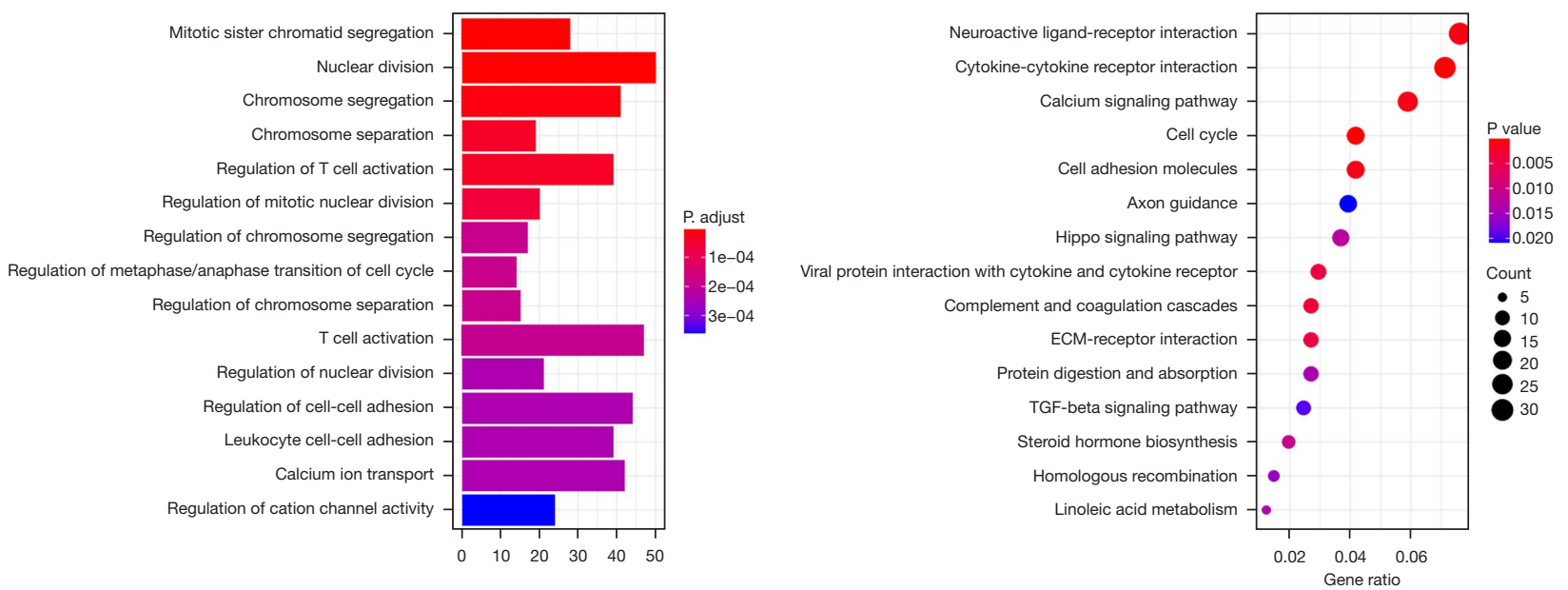

Figure 2 Construction of a miRNA network for BIRC5 and key pathways related to the prognosis of ccRCC. ccRCC, clear cell renal cell carcinoma.

BIRC5 expression in ccRCC, the siRNAs for SNHG3 and LINC00997 were separately transfected into the A498 RCC cell lines. Surprisingly, in A498 cells, LINC00997 siRNA did not significantly down-regulate BIRC5 expression, whereas SNHG3 siRNA dramatically inhibited BIRC5 expression (Figure $5 A$ ). From this, we can infer that lncRNA SNHG3 modulated BIRC5 protein expression. Results of Transwell migration and wound-healing assays also showed that SNHG3 siRNA successfully prohibited the migration and invasion of A498 and 786-O cells (Figure 5B,5C). Furthermore, dual-luciferase reporter assay confirmed that
miR-10b-5p mimics remarkably decreased the luciferase activity in SNHG3-WT-transfected A498 and 786-O cells (Figure 5D). The above results confirmed that lncRNA SNHG3 promotes ccRCC progression by upregulating BIRC5 expression via sponging hsa-miR-10b-5p.

\section{Discussion}

Clear cell RCC is one of the most common malignancies globally, and its progression is a complicated process. Therefore, it is vital to uncover the mechanism underlying 

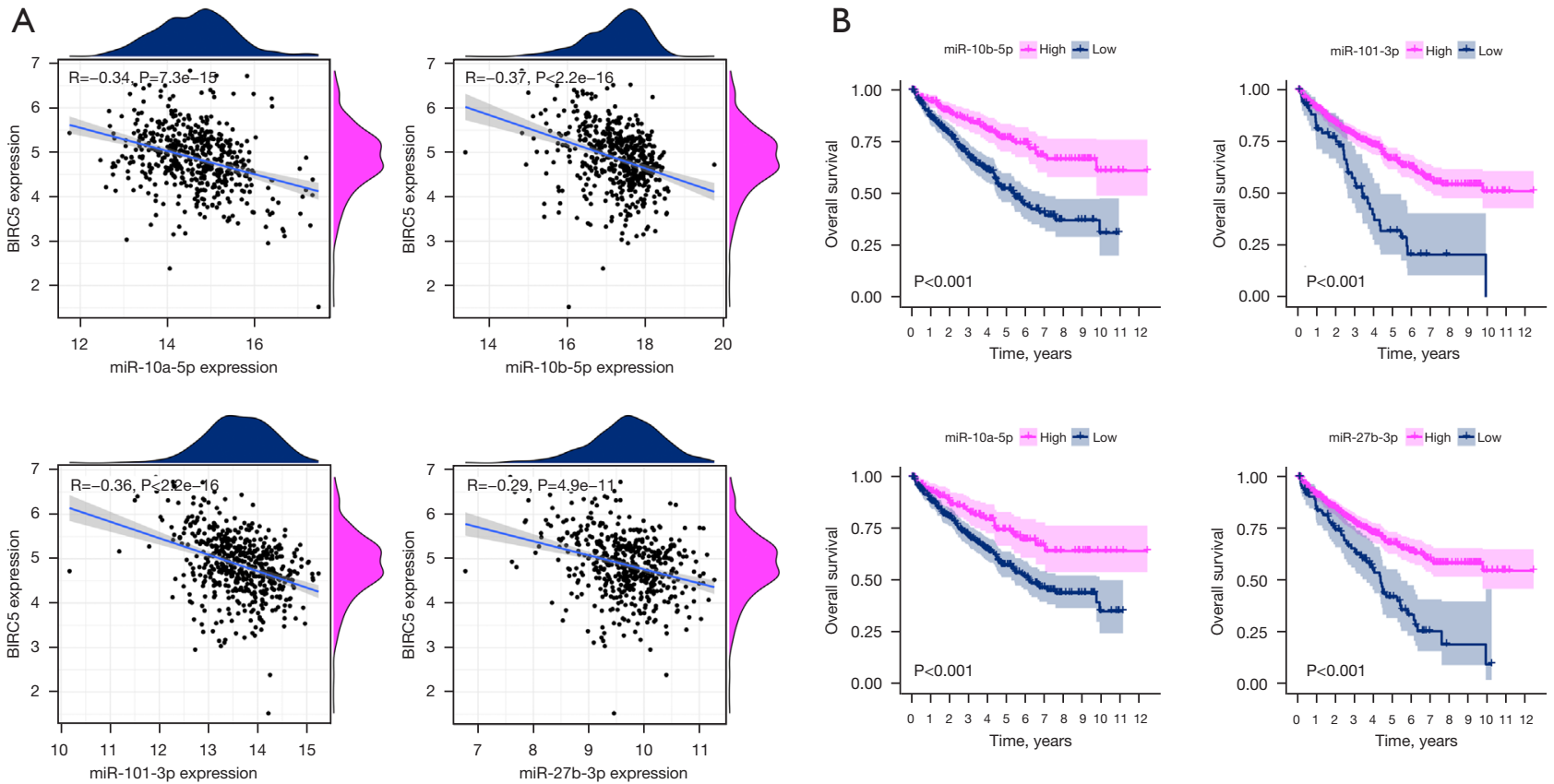

Figure 3 Four miRNAs (miR-101-3p, miR-10b-5p, miR-27b-3p, and miR-10a-5p) are negatively correlated with BICR5 gene expression. (A) Correlation analysis of all the miRNAs in Figure 2; only four microRNAs (miR-101-3p, miR-10b-5p, miR-27b-3p, and miR-10a-5p) are negatively correlated with BICR5 gene expression. (B) Survival analysis of all the miRNAs in Figure 2; only four miRNAs are significantly correlated with ccRCC prognosis. miRNA, microRNA; ccRCC, clear cell renal cell carcinoma.

ccRCC progression. Mounting evidence shows that ccRCC progression is closely related to abnormal lncRNA expression. For instance, Hu et al. found that the lncRNA MSC-AS1 modulated the proliferation and migration of ccRCC cells via a miR-3924/WNT5A axis (15). Another study reported that IncRNA SARCC suppressed ccRCC progression via altering the androgen receptor (AR)/ miRNA-143-3p axis (16). However, the mechanisms by which lncRNAs influence ccRCC are largely unknown.

In the present study, through analysis using the ENCORI database, we found that both lncRNA SNHG3 and LINC00997 are remarkably overexpressed and negatively correlated with the OS of patients with ccRCC. At a functional level, these two lncRNAs may facilitate ccRCC progression by upregulating BIRC5 expression. BIRC5, as a vital anti-apoptotic protein, participates in the progression of many cancers by promoting cell proliferation. Our research found that the BIRC5 gene was highly expressed in 33 malignancies, and we also validated high BIRC5 mRNA expression levels in ccRCC tissues via PCR, which corresponds with the results of other studies (17). In our study, we further demonstrated that silencing SNHG3 with siRNA could successfully prohibit BIRC5 protein expression in A498 cells. However, LINC00997 siRNA could not significantly inhibit BIRC5 protein expression in the A498 cells, which can be explained by the fact that LINC00997 contributes to ccRCC metastasis via regulating S100A11 protein expression (8). Most importantly, we confirmed that silencing SNHG3 could inhibit the migration and invasion of the ccRCC cells including the A498 and, 786-O.

Mounting evidence confirms that lncRNAs promote tumorigenesis via sponging miRNAs to promote tumorrelated protein expression $(18,19)$. miRNAs modulate various cellular processes via their molecular targets (20). Li et al. reported that miR-10b-5p was significantly lowly expressed in RCC and that its mimics inhibited cell proliferation (21). By using the luciferase reporter assay, we discovered that lncRNA SNHG3 binds to miR-10b-5p in ccRCC. Together, these data suggest that lncRNA SNHG3 exerts its oncogenic activity via the miR-10b-5p/BIRC5 axis.

The relevant characteristics of the tumor microenvironment of ccRCC are the interaction of tumor cells with surrounding tissues such as the cancer-associated fibroblasts (CAFs) and immune cells (22). Li et al. reported SNHG3 was also higher expression in the CAFs (23). Therefore, SNHG3 may higher in the CAFs and possibly be transferred to ccRCC via 
A

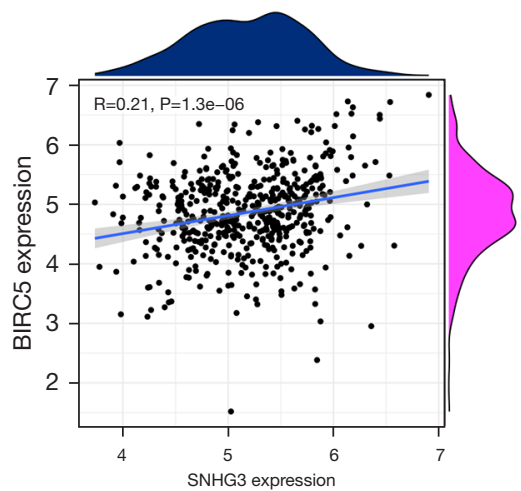

$\mathrm{D}$



G

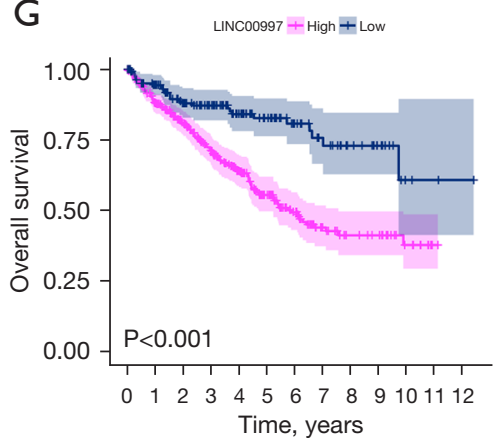

B

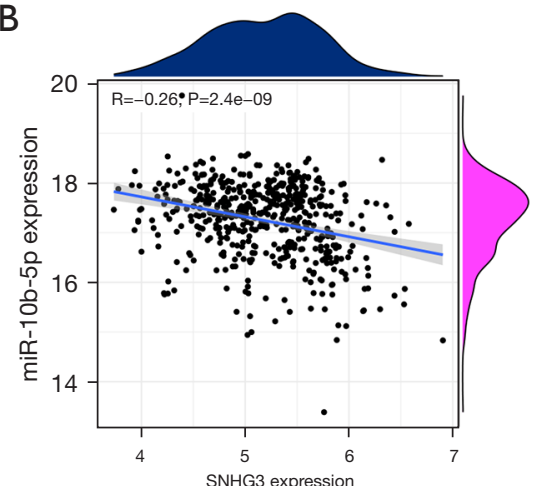

E

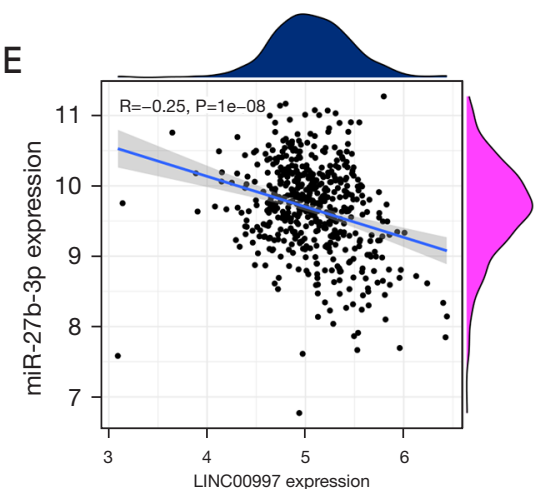

$\mathrm{H}$

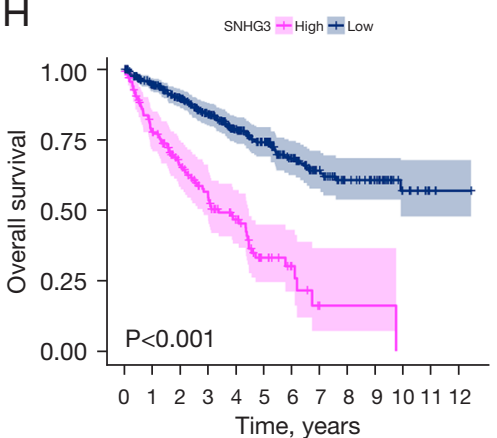

C



$\mathrm{F}$

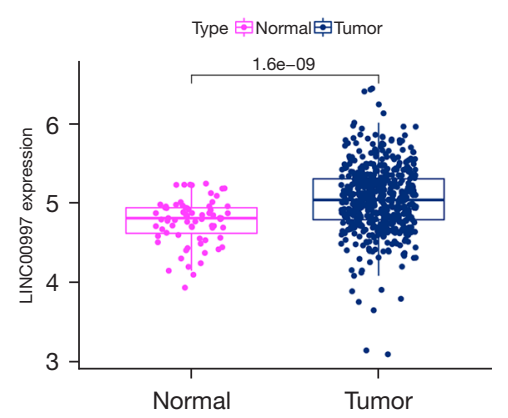

I

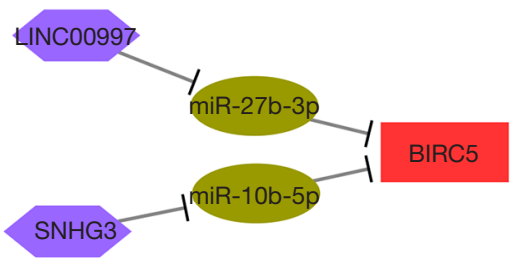

Figure 4 The constructed lncRNA-miRNA-BIRC5 network is associated with ccRCC progression. (A) Correlation analysis between SNHG3 and BIRC5. (B) Correlation analysis between SNHG3 and hsa-miR-10b-5p. (C) Expression level of SNHG3 in patients with ccRCC from TCGA. (D) Correlation analysis between LINC00997 and BIRC5. (E) Correlation analysis between LINC00997 and miR27b-3p. (F) Expression level of LINC00997 in patients with ccRCC from TCGA. (G) Overall survival analysis of LINC00997 in ccRCC patients from TCGA. (H) Overall survival analysis of SNHG3 in ccRCC patients from TCGA. (I) Regulatory ceRNA network for BIRC5. lncRNA, long non-coding RNA; miRNA, microRNA; ccRCC, clear cell renal cell carcinoma; TGCA, The Cancer Genome Atlas.

exosomes to regulate tumor microenvironment of ccRCC. Actually, In addition to SNHG3, immune related long noncoding RNAs (IRlncRs) including the lncRNA-IL10RBDT and MELTF-AS1 have been reported to affect immune status of the tumor microenvironment and were considered as the independent prognostic factor (24). In the future, small molecule tumor targeted therapy is the frontier of drug development; drugs for SNHG3 positive ccRCC IncRNAi will be developed. In addition, The autophagy-related lncRNAs were associated with the poor prognosis of ccRCC, Xuan et al. 

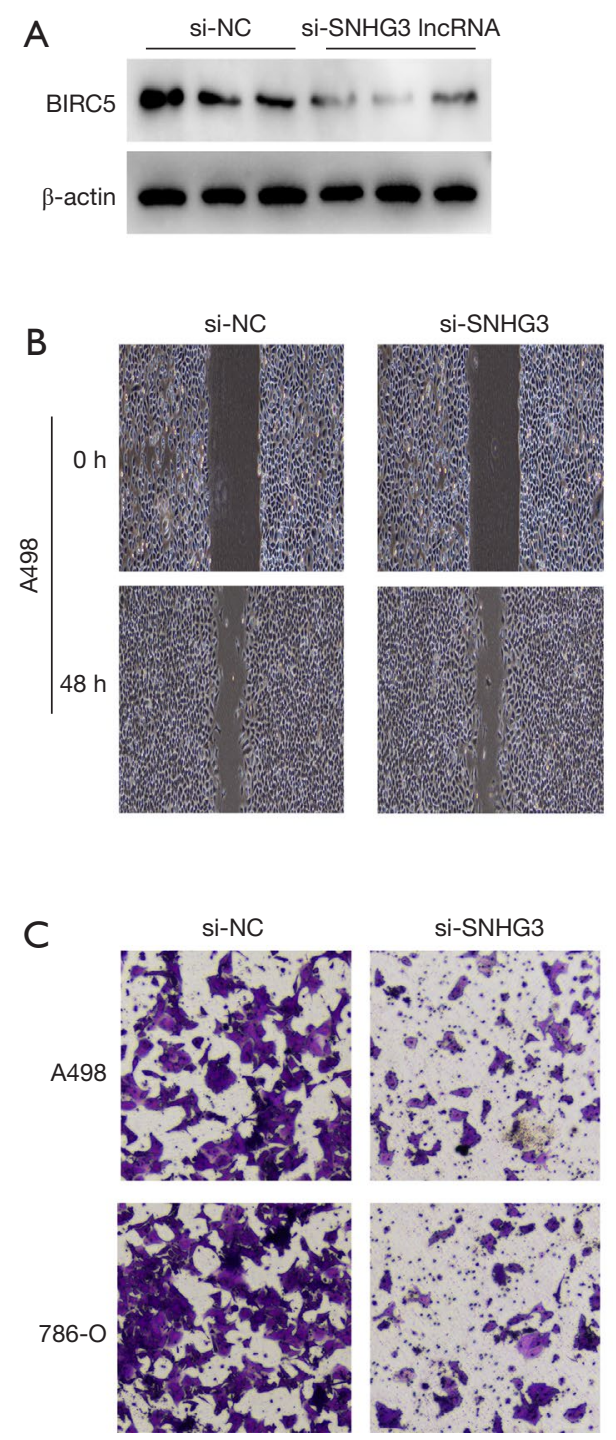
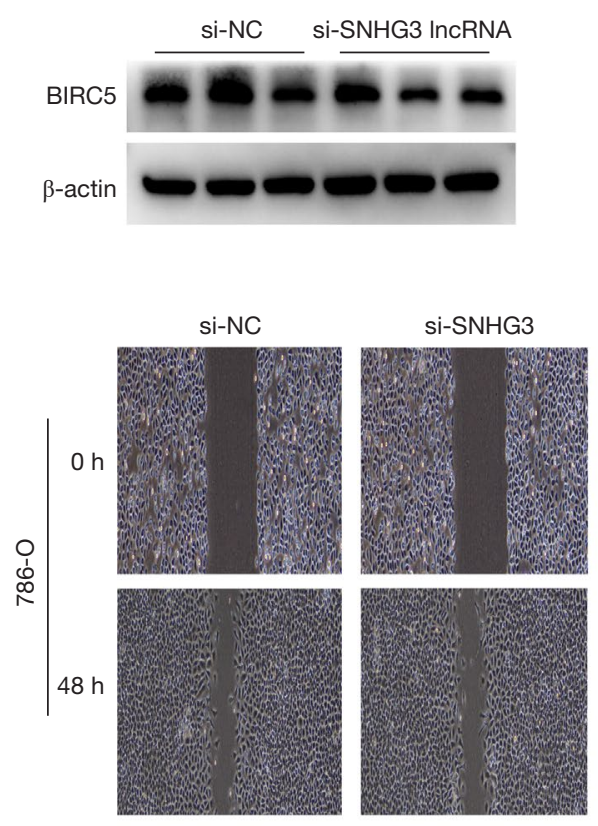

D



Figure 5 The lncRNA SNHG3/hsa-miR-10b-5p/BIRC5 axis is a vital pathway contributing to ccRCC progression. (A) The efficiency of SNHG3 knockdown in A498 cells transfected with SNHG3 siRNA was verified by western blot. (B,C) Migration (magnification 40x) and invasion (magnification 40×, 0.5\% crystal violet staining) assays for A498 and 786-O ccRCC cells transfected with SNHG3 siRNA. (D) Luciferase reporter assay was employed to examine A498 and 786-O cells transfected with miR-10b-5p mimics or miR-NC. **, P<0.01 vs. miR-NC group. lncRNA, long non-coding RNA; ccRCC, clear cell renal cell carcinoma; siRNA, small interfering RNA; NC, normal control.

team found seven autophagy-related lncRNAs including the SNHG3, SNHG17, MELTF-AS1, HOTAIRM1, EPB41L4ADT, AP003352.1, and AC145423.2 could be independently as a prognostic index for patients with ccRCC (25).

Although we have carried out cell experimental research in combination with bioinformatics, there are still some limitations in the study. Firstly, we didn't conduct the experiment of SNHG3-related ccRCC proliferation. We can refer to Zhang et al. team found that SNHG3 promotes ccRCC proliferation (7). Secondly, SNHG3 was not confirmed as a potential biomarker for patient risk stratification and local regional metastasis in ccRCC. Actually, SNHG3 as an oncogene has been used as a potential biomarker for patient risk stratification in multiple 
tumour types including the ovarian cancer, breast cancer, lung cancer (26-28). In addition, higher SNHG3 in the tumor tissues have the more lymph node metastasis such as the papillary thyroid carcinoma and breast cancer $(27,29)$. Most important, Jiang et al. team preformed metaanalysis and review to conclude that higher SNHG3 is closely related to with patient risk stratification and local regional metastasis in various tumors (30). The core of our research is to construct lncRNA network and found higher SNHG3 has the poor survival. From this, we can infer SNHG3 also could be used as a potential biomarker for patient risk stratification and local regional metastasis in ccRCC. Thirdly, the SNHG3 related to ccRCC in vivo was not conducted. Most evidence showed that knockdown of lncRNA SNHG3 inhibited the tumour growth including the bladder cancer and gastric cancer $(31,32)$. In summary, knockdown SNHG3 have the inhibitory role of ccRCC in vivo. The roles of SNHG3 in ccRCC in vivo need to be explored in future studies.

\section{Conclusions}

This study has shown that lncRNA SNHG3 is associated with a poor prognosis of ccRCC. Our findings also demonstrate that lncRNA SNHG3 boosts the proliferation, invasion, and migration abilities of ccRCC cells via sponging miR-10b-5p to upregulate BIRC5 expression. From the perspective of translational medicine, these data show that the IncRNA SNHG3/miR-10b-5p/BIRC5 axis could be a therapeutic target for the management of ccRCC.

\section{Acknowledgments}

Funding: This study was supported by Lishui City People's Hospital Step Ladder project (grant No. 2017CTB010).

\section{Footnote}

Reporting Checklist: The authors have completed the MDAR reporting checklist. Available at https://dx.doi. org/10.21037/tcr-21-1802

Data Sharing Statement: Available at https://dx.doi. org/10.21037/tcr-21-1802

Conflicts of Interest: All authors have completed the ICMJE uniform disclosure form (available at https://dx.doi. org/10.21037/tcr-21-1802). The authors have no conflicts of interest to declare.

Etbical Statement: The authors are accountable for all aspects of the work in ensuring that questions related to the accuracy or integrity of any part of the work are appropriately investigated and resolved. The study was conducted in accordance with the Declaration of Helsinki (as revised in 2013). The study was approved by the Ethics Committee of Lishui People's Hospital (No.: LLW-F0-403) and informed consent was taken from all the patients.

Open Access Statement: This is an Open Access article distributed in accordance with the Creative Commons Attribution-NonCommercial-NoDerivs 4.0 International License (CC BY-NC-ND 4.0), which permits the noncommercial replication and distribution of the article with the strict proviso that no changes or edits are made and the original work is properly cited (including links to both the formal publication through the relevant DOI and the license). See: https://creativecommons.org/licenses/by-nc-nd/4.0/.

\section{References}

1. Gupta K, Miller JD, Li JZ, et al. Epidemiologic and socioeconomic burden of metastatic renal cell carcinoma (mRCC): a literature review. Cancer Treat Rev 2008;34:193-205.

2. Rini BI, Campbell SC, Escudier B. Renal cell carcinoma. Lancet 2009;373:1119-32.

3. Yuan C, Xiong Z, Shi J, et al. Overexpression of PPT2 Represses the Clear Cell Renal Cell Carcinoma Progression by Reducing Epithelial-to-mesenchymal Transition. J Cancer 2020;11:1151-61.

4. Lane BR, Kattan MW. Prognostic models and algorithms in renal cell carcinoma. Urol Clin North Am 2008;35:613-25; vii.

5. Jathar S, Kumar V, Srivastava J, et al. Technological Developments in lncRNA Biology. Adv Exp Med Biol 2017;1008:283-323.

6. Peng WX, Koirala P, Mo YY. LncRNA-mediated regulation of cell signaling in cancer. Oncogene 2017;36:5661-7.

7. Zhang C, Qu Y, Xiao H, et al. LncRNA SNHG3 promotes clear cell renal cell carcinoma proliferation and migration by upregulating TOP2A. Exp Cell Res 
2019;384:111595.

8. Chang Y, Li N, Yuan W, et al. LINC00997, a novel long noncoding RNA, contributes to metastasis via regulation of S100A11 in kidney renal clear cell carcinoma. Int J Biochem Cell Biol 2019;116:105590.

9. Varkonyi-Gasic E, Hellens RP. qRT-PCR of Small RNAs. Methods Mol Biol 2010;631:109-22.

10. Wang Y, Du J, Niu X, et al. MiR-130a-3p attenuates activation and induces apoptosis of hepatic stellate cells in nonalcoholic fibrosing steatohepatitis by directly targeting TGFBR1 and TGFBR2. Cell Death Dis 2017;8:e2792.

11. Li JH, Liu S, Zhou H, et al. starBase v2.0: decoding miRNA-ceRNA, miRNA-ncRNA and protein-RNA interaction networks from large-scale CLIP-Seq data. Nucleic Acids Res 2014;42:D92-7.

12. Shannon P, Markiel A, Ozier O, et al. Cytoscape: a software environment for integrated models of biomolecular interaction networks. Genome Res 2003;13:2498-504.

13. Mejias M, Gallego J, Naranjo-Suarez S, et al. CPEB4 Increases Expression of PFKFB3 to Induce Glycolysis and Activate Mouse and Human Hepatic Stellate Cells, Promoting Liver Fibrosis. Gastroenterology 2020;159:273-88.

14. Troiano G, Guida A, Aquino G, et al. Integrative Histologic and Bioinformatics Analysis of BIRC5/Survivin Expression in Oral Squamous Cell Carcinoma. Int J Mol Sci 2018;19:2664.

15. Hu Z, Li L, Cheng P, et al. lncRNA MSC-AS1 activates $\mathrm{Wnt} / \beta$-catenin signaling pathway to modulate cell proliferation and migration in kidney renal clear cell carcinoma via miR-3924/WNT5A. J Cell Biochem 2020;121:4085-93.

16. Zhai W, Sun Y, Guo C, et al. LncRNA-SARCC suppresses renal cell carcinoma (RCC) progression via altering the androgen receptor(AR)/miRNA-143-3p signals. Cell Death Differ 2017;24:1502-17.

17. Wang J, Chen M, Dang C, et al. The Early Diagnostic and Prognostic Value of BIRC5 in Clear-Cell Renal Cell Carcinoma Based on the Cancer Genome Atlas Data. Urol Int 2021. [Epub ahead of print].

18. Kong X, Duan Y, Sang Y, et al. LncRNA-CDC6 promotes breast cancer progression and function as ceRNA to target CDC6 by sponging microRNA-215. J Cell Physiol 2019;234:9105-17.

19. Lu J, Xu F, Lu H. LncRNA PVT1 regulates ferroptosis through miR-214-mediated TFR1 and p53. Life Sci 2020;260:118305.

20. Croce CM, Calin GA. miRNAs, cancer, and stem cell division. Cell 2005;122:6-7.

21. Li Y, Chen D, Li Y, et al. Oncogenic cAMP responsive element binding protein 1 is overexpressed upon loss of tumor suppressive miR-10b-5p and miR-363-3p in renal cancer. Oncol Rep 2016;35:1967-78.

22. Wolf MM, Kimryn Rathmell W, Beckermann KE. Modeling clear cell renal cell carcinoma and therapeutic implications. Oncogene 2020;39:3413-26.

23. Li Y, Zhao Z, Liu W, et al. SNHG3 Functions as miRNA Sponge to Promote Breast Cancer Cells Growth Through the Metabolic Reprogramming. Appl Biochem Biotechnol 2020;191:1084-99.

24. Jiang Y, Gou X, Wei Z, et al. Bioinformatics profiling integrating a three immune-related long non-coding RNA signature as a prognostic model for clear cell renal cell carcinoma. Cancer Cell Int 2020;20:166.

25. Xuan Y, Chen W, Liu K, et al. A Risk Signature with Autophagy-Related Long Noncoding RNAs for Predicting the Prognosis of Clear Cell Renal Cell Carcinoma: Based on the TCGA Database and Bioinformatics. Dis Markers 2021;2021:8849977.

26. Hong L, Chen W, Wu D, et al. Upregulation of SNHG3 expression associated with poor prognosis and enhances malignant progression of ovarian cancer. Cancer Biomark 2018;22:367-74.

27. Ma Q, Qi X, Lin X, et al. LncRNA SNHG3 promotes cell proliferation and invasion through the miR-384/ hepatoma-derived growth factor axis in breast cancer. Hum Cell 2020;33:232-42.

28. Shi J, Li J, Yang S, et al. LncRNA SNHG3 is activated by $\mathrm{E} 2 \mathrm{~F} 1$ and promotes proliferation and migration of nonsmall-cell lung cancer cells through activating TGF- $\beta$ pathway and IL-6/JAK2/STAT3 pathway. J Cell Physiol 2020;235:2891-900.

29. Sui G, Zhang B, Fei D, et al. The lncRNA SNHG3 accelerates papillary thyroid carcinoma progression via the miR-214-3p/PSMD10 axis. J Cell Physiol 2020;235:6615-24.

30. Jiang Y, Le L. Overexpression of lncRNA SNGH3 Predicts Unfavorable Prognosis and Clinical Outcomes in Human Cancers: Evidence from a Meta-Analysis. Biomed Res Int 2020;2020:7974034.

31. Dai G, Huang C, Yang J, et al. LncRNA SNHG3 
promotes bladder cancer proliferation and metastasis through miR-515-5p/GINS2 axis. J Cell Mol Med 2020;24:9231-43.

32. Sun B, Han Y, Cai H, et al. Long non-coding RNA SNHG3, induced by IL-6/STAT3 transactivation,

Cite this article as: $\mathrm{Xu} \mathrm{Z,} \mathrm{Ye} \mathrm{J,} \mathrm{Bao} \mathrm{P,} \mathrm{Wu} \mathrm{Q,} \mathrm{Xie} \mathrm{F,} \mathrm{Li} \mathrm{P.}$ Long non-coding RNA SNHG3 promotes the progression of clear cell renal cell carcinoma via regulating BIRC5 expression. Transl Cancer Res 2021;10(10):4502-4513. doi: $10.21037 /$ tcr-21-1802 promotes stem cell-like properties of gastric cancer cells by regulating the miR-3619-5p/ARL2 axis. Cell Oncol (Dordr) 2021;44:179-92.

(English Language Editor: J. Reynolds) 